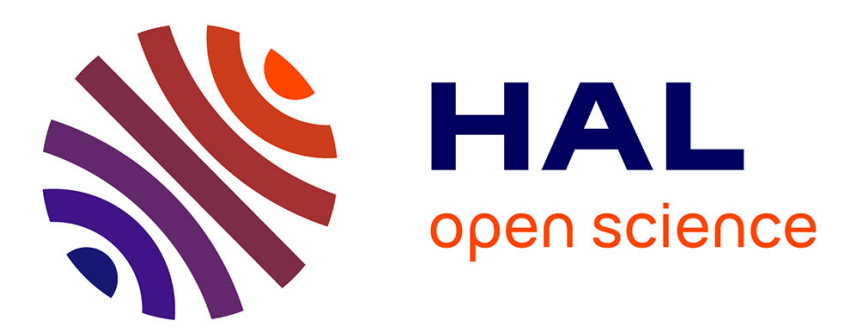

\title{
Seismic responses to fluid pressure perturbations in a slipping fault,
}

B. Derode, Y. Guglielmi, F. Cappa, L. de Barros

\section{To cite this version:}

B. Derode, Y. Guglielmi, F. Cappa, L. de Barros. Seismic responses to fluid pressure perturbations in a slipping fault,. Geophysical Research Letters, 2015, pp.3197-3203. 10.1002/2015GL063671 . hal-01183356

\section{HAL Id: hal-01183356 https://hal.science/hal-01183356}

Submitted on 12 Nov 2021

HAL is a multi-disciplinary open access archive for the deposit and dissemination of scientific research documents, whether they are published or not. The documents may come from teaching and research institutions in France or abroad, or from public or private research centers.
L'archive ouverte pluridisciplinaire HAL, est destinée au dépôt et à la diffusion de documents scientifiques de niveau recherche, publiés ou non, émanant des établissements d'enseignement et de recherche français ou étrangers, des laboratoires publics ou privés.

$$
\text { Copyright }
$$




\section{Geophysical Research Letters}

\author{
RESEARCH LETTER \\ 10.1002/2015GL063671 \\ Key Points: \\ - Synchronous seismic and \\ hydromechanical measurements \\ are made in situ in a fault \\ - Microearthquakes, long period \\ and tremor-like signal events \\ were recorded \\ - These three types of event are \\ associated to various hydromechanica \\ processes
}

Supporting Information:

- Text S1

Correspondence to:

B. Derode,

benoit@dgf.uchile.cl

\section{Citation:}

Derode, B., Y. Guglielmi, L. De Barros, and F. Cappa (2015), Seismic responses to fluid pressure perturbations in a slipping fault, Geophys. Res. Lett., 42, 3197-3203, doi:10.1002/2015GL063671.

Received 10 MAR 2015 Accepted 2 APR 2015

Accepted article online 7 APR 2015 Published online 5 MAY 2015
○2015. American Geophysical Union. All Rights Reserved.

\section{Seismic responses to fluid pressure perturbations in a slipping fault}

\author{
Benoit Derode $^{1,2}$, Yves Guglielmi ${ }^{3}$, Louis De Barros ${ }^{2}$, and Frédéric Cappa ${ }^{2}$ \\ ${ }^{1}$ Department of Geophysics, University of Chile, Santiago, Chile, ${ }^{2}$ Géoazur (UMR 7329), University of Nice Sophia-Antipolis, \\ CNRS, IRD, Côte d'Azur Observatory, Nice, France, ${ }^{3}$ CEREGE (UMR 7330), University of Aix-Marseille, Marseille, France
}

\begin{abstract}
Seismicity induced by fluid injection in a natural fault is investigated in situ in the near field of the source. We present synchronous seismic and hydromechanical measurements directly recorded in the decametric injection zone. The three main types of seismic events were recorded during injection and shut-in: high-amplitude and short duration seismic events (SE) (i.e., microearthquakes), low to constant amplitude and 5 to $17 \mathrm{~s}$ long tremor-like signals (TLS), and long period events (LP) with a narrow-frequency band content. Seismicity first initiates with a sequence of SE and TLS, when pressure is high ( 3.5 MPa), slip is activated on the fault, which experiences a twentyfold increase of permeability. Then LP events appear to be associated to fluid leakage in the fault caused by dilation during slip. During shut-in, residual pressures as low as 0.6 MPa still trigger SE events. We show that the initial TLS sequence triggers when a progressive transition occurs from rupture controlled by effective stress variations close to the injection source to a large friction weakening-dominated slip on the fault. We conclude that the combination of these different seismic signal types may be a proxy to monitor fault instability associated to fluid pressure perturbations.
\end{abstract}

\section{Introduction}

Fluids are known to be a triggering and increasing factor of the rupture in seismogenic faults [Sibson, 1982; Muir-Wood and King, 1993]. The most common feature is the nucleation of seismic rupture along a slip surface by localized high-fluid pressures that reduce the effective stress and the shear strength [Scholz, 2002]. Such rupture is the final stage of a series of steps that begin with the initiation and growth of local microcracks and fracture planes that, with increased stress and fluid pressure, eventually coalesce to form a continuous slip surface. This initiation phase can be associated with a series of small seismic events [Amitrano et al., 2010; Bouchon et al., 2011]. If the effects of fluid pressures in the concept of stable and unstable sliding are known on theoretical grounds, experimental data to constrain those theories are rare [Cornet et al., 1997; Guglielmi et al., 2008; Cornet, 2012], and relating observations with theory is challenging.

The fluid effects in the rupture process are commonly interpreted as linked to the appearance of unusual seismicity (e.g., Long Period events (LP, also called Low Frequency, LF, events) and Tremors-Like Signals (TLS)) that was observed in different geological contexts, such as volcanoes [Chouet, 2003], reservoir rocks [Das and Zoback, 2011], subduction zones and crustal faults [Obara, 2002; Ide et al., 2007], or yet landslides [Peng and Gomberg, 2010]. For instance, TLS are recognized as the seismic signals associated with slow-slip stress release on seismogenic faults, and they might be seen as potential signature of the nucleation of large earthquakes [Zigone et al., 2011; Peng and Gomberg, 2010]. Moreover, tremors are often thought to be associated with high-fluid pressures at depth that have a notable weakening effect on the fault properties [Shelly et al., 2006; Miyazawa and Brodsky, 2008; Becken et al., 2011]. However, despite these numerous observations connecting fluid pressures and seismicity, the source processes of this seismicity is still questionable, and the role of fluids in the rupture process remains unclear. When faults are fluid flow paths, a first challenge is to understand how the fluid pressure modifies the medium properties and the stresses before driving the fault to rupture. A second challenge is to characterize the seismicity associated with the hydraulic perturbations.

In this paper, we present synchronous measurements of fluid pressure and seismicity recorded in the near field of a fluid injection into a fault along which slip is induced on a patch of $\sim 10 \mathrm{~m}$ in size at $282 \mathrm{~m}$ depth in the Low Noise Underground Laboratory in France (http://lsbb.oca.eu) (Figure 1). Thanks to the proximal monitoring (meters) at high frequency $(\sim \mathrm{kHz})$, the links between the different seismic responses associated with transient fluid pressures and fault deformation can be investigated. During the $2200 \mathrm{~s}$ injection, the fluid 


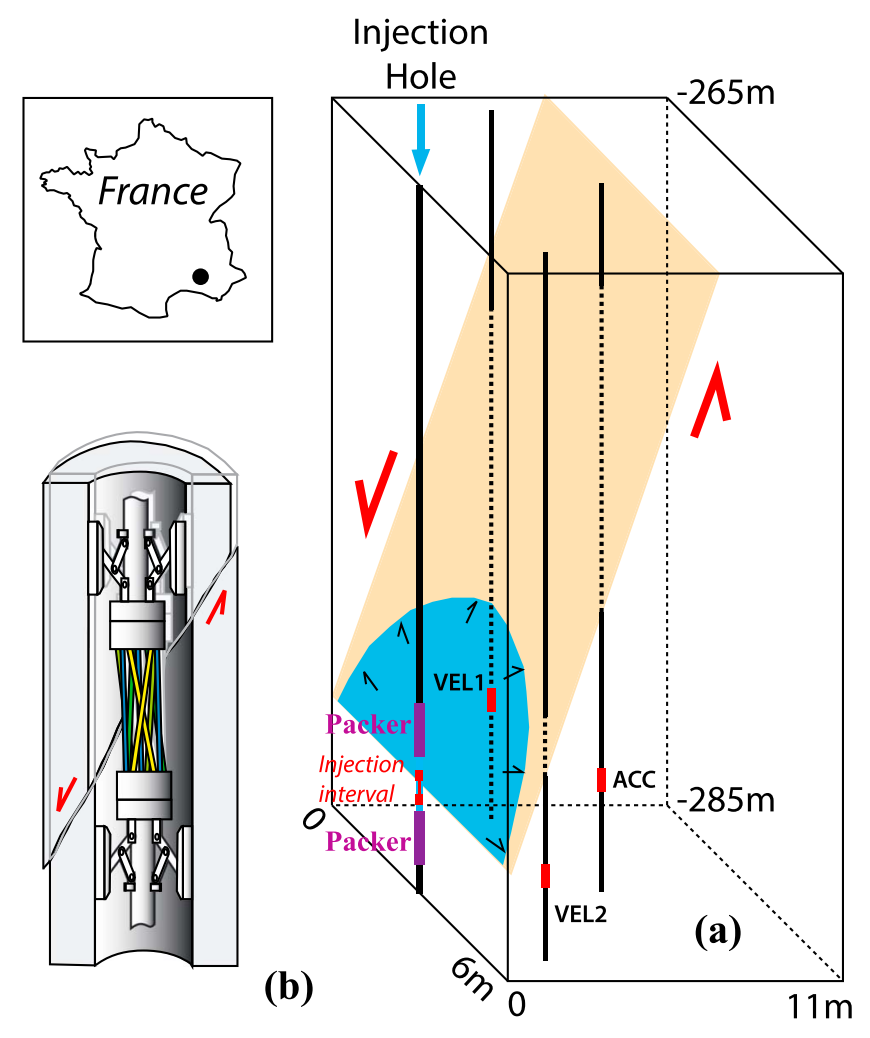

Figure 1. Experimental setup: (a) location of the boreholes instrumentation at $282 \mathrm{~m}$ depth across the fault plane (represented in orange); (b) detail view of the displacement sensor that captured the fault slip in the injection chamber (see additional material). pressure was progressively increased step by step and maintained to $3.5 \mathrm{MPa}$, and then decreased. Our observations reveal distinct behaviors, including poroelastic fault movements at the beginning of the pressure increase, followed by aseismic slip from the middle of the pressure increase phase to the maximum pressure, and, finally, seismic rupture starting toward the middle of pressure peak phase and continuing during shut-in. Thanks to the seismological monitoring, this deformation sequence was related to three distinct families of seismic signals during the fault rupture: impulsive, high-frequency events (i.e., microearthquakes), tremor-like signals, and low-frequency events, each of them appearing at different time of the fluid pressure evolution.

\section{Experiment Setup}

The experiment aims at injecting highpressure water in a fault zone in order to induce a seismic reactivation at moderate level (i.e., slip of about a millimeter). Four boreholes, the injection hole and three seismic monitoring holes, respectively, were drilled vertically through a segment of the fault zone intersecting limestones. Boreholes are spaced of about 2-3 m horizontally (Figure 1). The fault zone has an orientation of $\mathrm{N} 030 \mathrm{E}$, a dip angle of $\sim 70^{\circ}$, and a mean length of $\sim 100 \mathrm{~m}$. The fault has an average initial permeability of $7 \times 10^{-12} \mathrm{~m}^{2}$ and a bulk modulus of 10 to $17 \mathrm{GPa}$ that are a factor 25 higher and a factor of 2 to 5 lower than the surrounding host rock, respectively [Jeanne et al., 2012; Derode et al., 2013]. The change in fluid pressure in the fault is controlled by a step-rate water injection applied into a $1.2 \mathrm{~m}$ long sealed section isolated between two inflatable packers. The pressure and flow rate variations range from 0 to $3.5 \mathrm{MPa}$ and from 0 to $1.01 \times 10^{-3} \mathrm{~m}^{3} \mathrm{~s}^{-1}$, respectively. The total amount of injected water is $1.286 \mathrm{~m}^{3}$. During the injection, we continuously measured the fluid pressure and the injection rate at a sampling frequency of $1 \mathrm{~Hz}$. Fault slip was measured using a fiber-optic deformation sensor (three components) set in the injection hole across the fault [Guglielmi et al., 2014] (Figure 1b and additional material). Synchronously, the seismic emissions were recorded at $8 \mathrm{kHz}$ by a set of 1 three component accelerometer (Acc: KB12VB, 0.15-260 Hz, Figure 1a) and 2 three-component geophones (Vel1: GS11D, 4.5-100 Hz and Vel2: GS20DH, 40-500 Hz, Figure 1a). These sensors are spaced horizontally from 3 to $5 \mathrm{~m}$ of the injection point and are at the same depth than injection. These three sensors cover a large sampling band and thus allow for a precise distinction of seismic events in the near field of the injection.

\section{Seismic Events Type and Sequence Generated by Injection}

Data indicate that almost no seismicity was recorded during the pressure increasing steps from 1 to 14 (Figure 2). The seismic emissions started at the middle of the maximum pressure plateau, between steps 14 and 16. The seismicity then lasted all along the pressure decrease until the end of injection at a relatively low residual pressure of $0.65 \mathrm{MPa}$ (step 21). More than 200 seismic events were recorded in less than $20 \mathrm{~min}$. Most of the events are triggered during shut-in and not during the increasing pressure phase. The seismicity rate (15 events/min) is roughly constant all along the seismic emission period. 


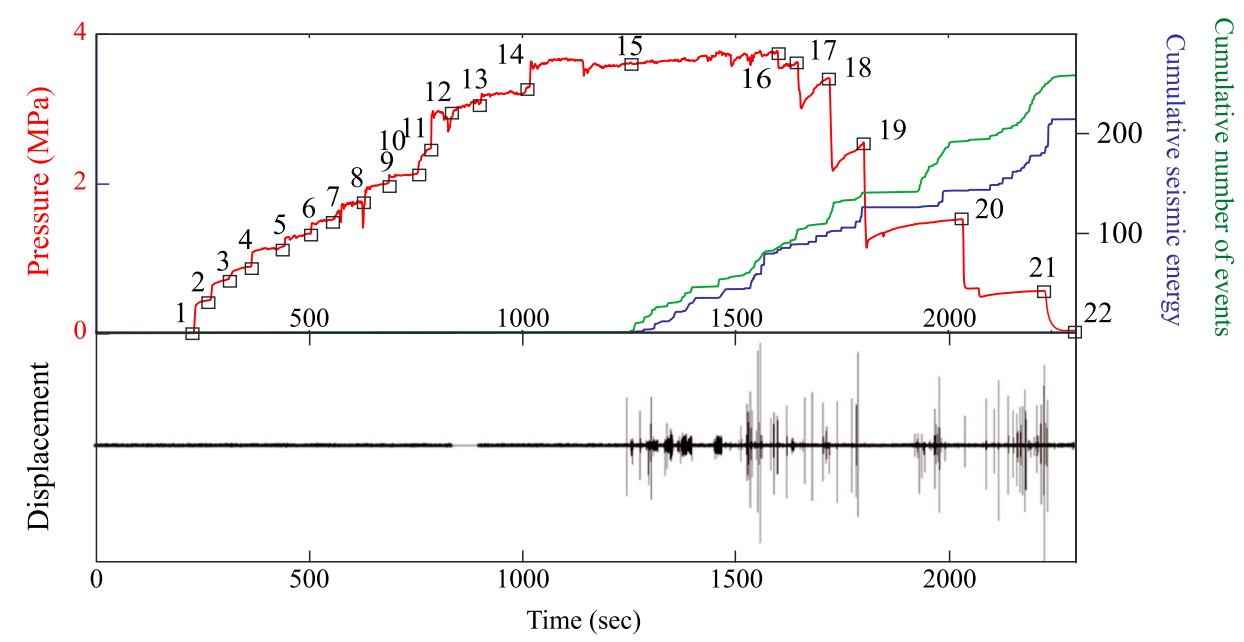

Figure 2. (top) Variations of the fluid pressure (in red) during the injection (numbers correspond to the imposed pressure steps except number 15 which marks the onset of seismicity), cumulative number of seismic events (in green) extracted using a threshold applied on the seismic energy, and cumulative seismic energy (in blue). (top) Seismic signals recorded by the $Z$ component of Vel2 geophone (see location in Figure 1a).

In Figure $2 b$, the different types of events induced by injection are presented. Events are extracted using a threshold value applied on the seismic energy using the $Z$ component of the "Vel2" sensor. We then checked that extracted events are coherent from one sensor to each other. Based on their waveforms, duration and frequency contents, we separated the events in three main classes (Figures 3a-3c): tectoniclike seismic events (SE) (i.e., microearthquakes), long period events (LP), and tremor-like signals (TLS). The SE events are typically characterized by an impulsive onset, a short duration (less than $0.1 \mathrm{~s}$ ) and high amplitude (Figure 3a). Energy is distributed on the entire frequency band. LP events have small amplitude and a short duration (Figure 3b). They mainly differ from the SE by their frequency content, as they have a narrower-frequency band, with energy only in the $250-450 \mathrm{~Hz}$ band. The TLS events are characterized by an extended duration up to 200 times the SE events, as they last up to $17 \mathrm{~s}$ (Figure 3c). TLS are characterized by (1) a progressive emergence from the background noise with a few single events before, (2) a long and complex signal at nearly constant amplitude, and (3) an abrupt termination. Frequency content is higher in the $200-500 \mathrm{~Hz}$ and $650-800 \mathrm{~Hz}$ bands.

The Fourier spectrum of the displacements of these three different types of seismic events is presented in Figure 3d. While SEs show a nearly flat response up to $500 \mathrm{~Hz}$, LP energy emerges from the noise level just in the 150-500 Hz band. The TLS events still have energy at low frequencies, even if most of their frequency content is above $200 \mathrm{~Hz}$. From 500 to $800 \mathrm{~Hz}$, the TLS and SEs have almost identical spectral contents, including the lack of energy around $600 \mathrm{~Hz}$. This means that the two frequency bands observed on the TLS events are likely due to the instrument responses and not to the signals themselves. Frequency corners are therefore at higher frequencies and cannot be computed.

Figure $3 e$ shows the timing of the seismic events with respect to the variations in fluid pressure. Although the seismicity started with a SE event, the first $250 \mathrm{~s}$ of signals are totally dominated by TLS emissions, with only few SEs. After that, no TLS are recorded and several swarms of SE occurred, associated with the increase of fluid pressure before step 15 and toward the end of every pressure step (Figure 2). The first swarm occurred when the fluid pressure was still high, but two thirds of the SEs were recorded during shut-in (after step 15). This suggests that, once the seismicity is starting, the failures in the medium seem to be self-maintaining. The LP events appear toward the end of the TLS period. Interestingly, these events seem to be associated with small fluid pressure drops and are present until the last pressure step (Figures 3e and 2).

\section{Discussion on Seismicity and Fault Hydromechanical Response}

The aim of our proximal seismological monitoring was to identify the potential seismic signatures during fluid injection directly in a fault. As only three seismic sensors, with their own frequency bands of response, were used, it is not possible to derive a full quantitative analysis of this seismicity, including source 

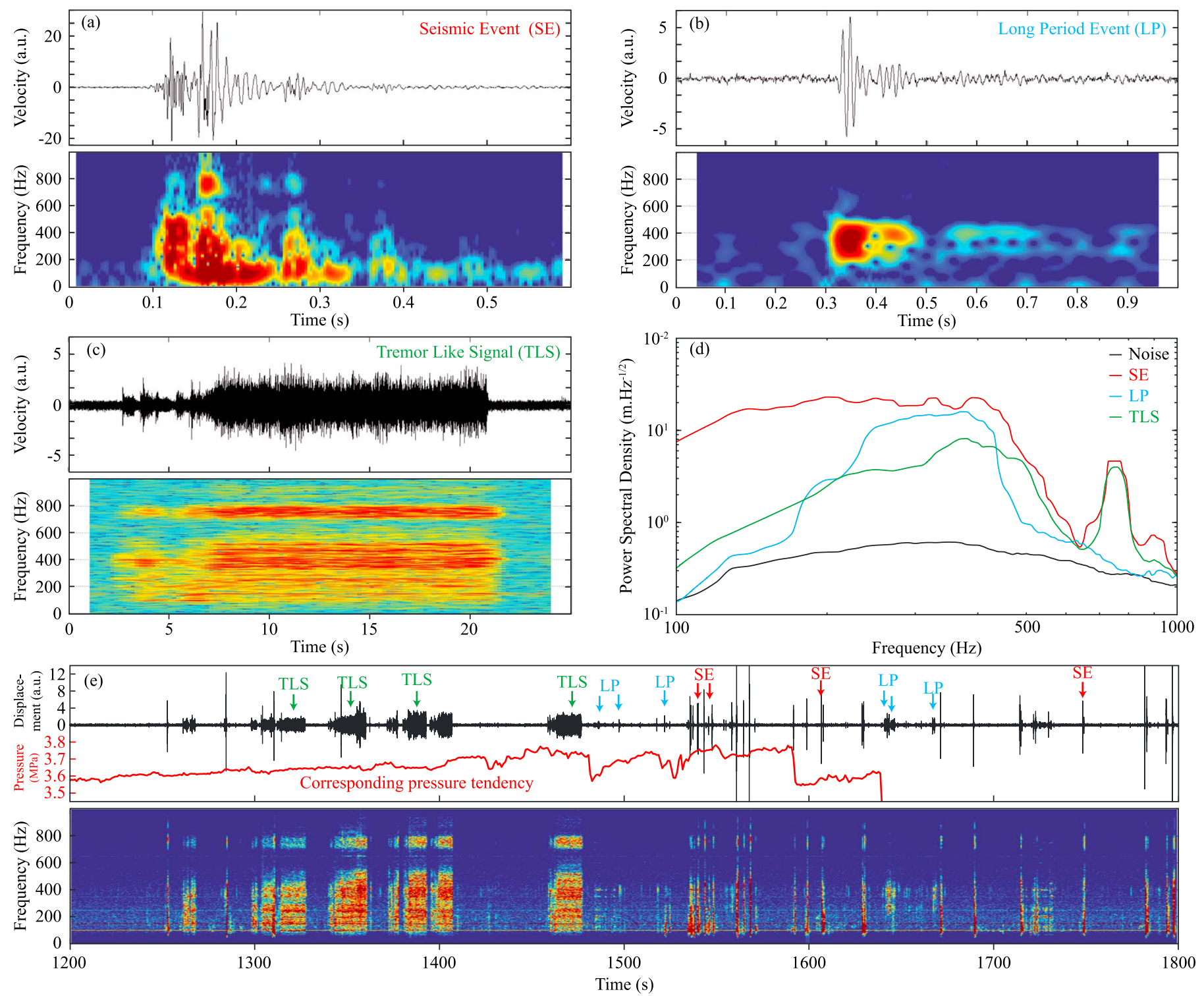

Figure 3. (a-c) Signals and spectrograms (Vel2, Z component) of the tectonic, TLS, and LP events, respectively. (d) Frequency spectrum smoothed using a median filter sliding windows of the displacement of the three types of seismic events and the background noise (displacement). (e) Pressure variations, seismic signals, and spectrogram in the 1200-1600 s time windows. Note that, on the spectrogram, the bands at frequencies multiple of $50 \mathrm{~Hz}$ are due to electrical noise.

properties (e.g., location and mechanisms). Moreover, as spurious frequency response appears as low as $600 \mathrm{~Hz}$, scaling laws such as frequency corner relationships cannot be investigated either. However, this experiment presents near-field measurements with unprecedented detail that reveal the complex behavior of a fault zone response to a local fluid pressure perturbation. Different types of seismic signals have been recorded, with specific signatures, which can be compared to ones observed on volcanoes [Chouet, 2003], reservoirs [Das and Zoback, 2013a, 2013b], seismogenic faults, and landslides [Peng and Gomberg, 2010]. The SEs especially show a classical earthquake behavior suggesting that they are generated by rapid slip along small $(<\mathrm{m})$ fractures. The frequency content is, however, much higher, with frequency corner above $600 \mathrm{~Hz}$, corresponding to the failure on submetric patches. On the other hand, the narrow-frequency bands of the LPs and their links with the fluid pressure drops might imply that these events are related to fluid processes in the volume surrounding the injection zone. They might be analogous with the LP events observed on volcanoes [Chouet, 2003] or in reservoirs [Tary et al., 2014] and commonly interpreted as fluid-filled cavity resonance. Recently, Das and Zoback [2011, 2013a, 2013b] 


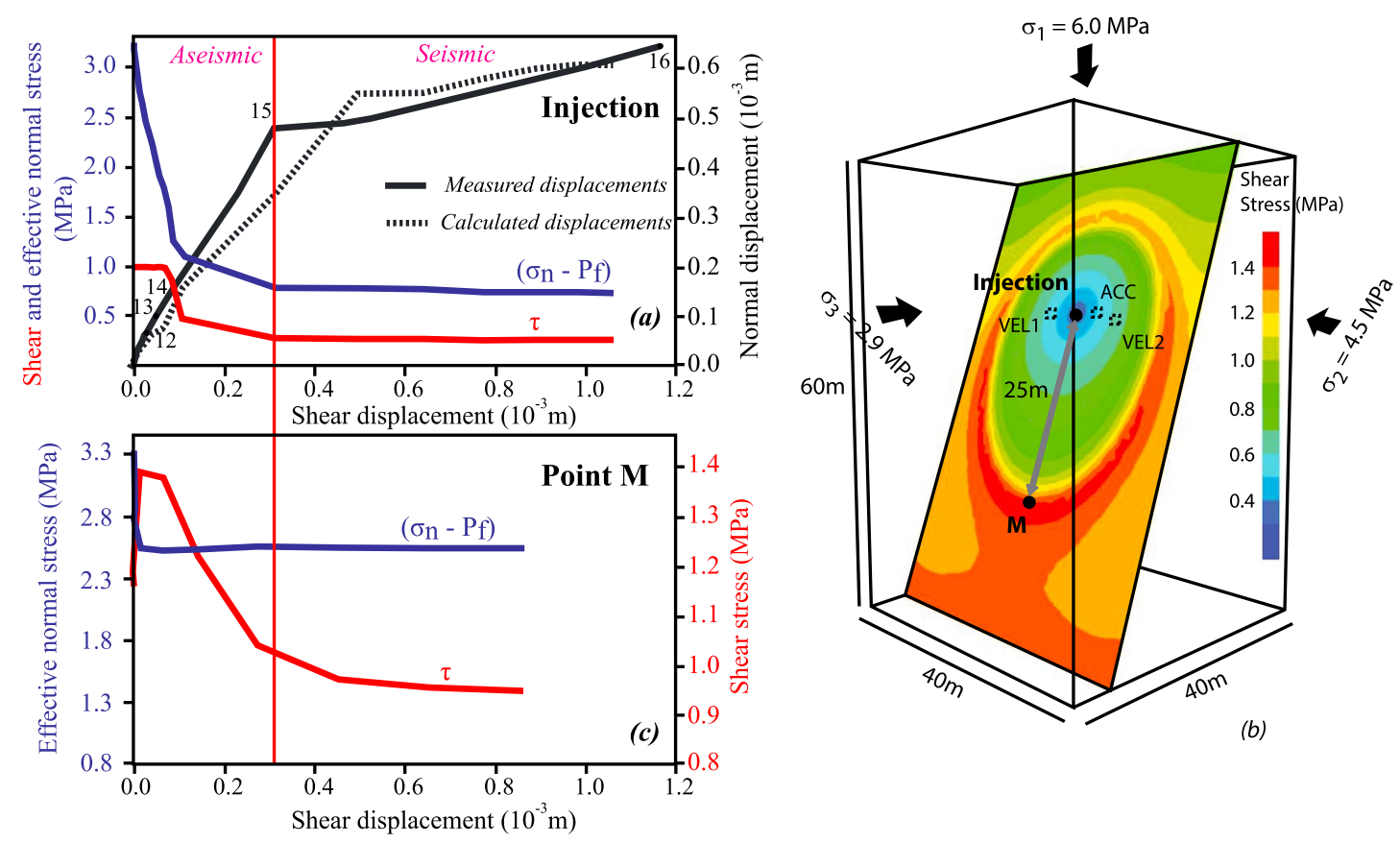

Figure 4. (a) Measured and calculated fault movements and stress at the injection point (from pressure step 12 to 16); (b) three-dimensional view of the fault with shear stress variation calculated at the onset of seismicity in the experiment (pressure step 15); and (c) calculated changes in effective normal and shear stress as function of fault slip at point $M$ reached by the pressurized patch at step 15.

observed tremor-like signals of long period and long duration during hydraulic stimulation of shale-gas reservoirs. These works show that these unusual events are remarkably similar in appearance to tectonic tremors observed in seismogenic zones [Das and Zoback, 2011]. Our signals also bear a striking resemblance even if the ruptures size in our experiment is much smaller, leading to a much higher frequency content of the seismicity (corner frequency above the spurious frequencies of the sensors). Unfortunately, the limited number of sensors and their low-frequency response prevent to compute accurately spectral properties of these events. Nevertheless, the lack of resonating patterns and the presence of energy in the very low frequency bands suggest that a fluid resonance process, such as observed on volcanoes [Chouet, 2003], is not suitable. On the contrary, the long duration and the waveforms of the TLS that we observed suggest that a mechanism of slow-slip failure might be viable. TLS signals might therefore be compared to tectonic tremors associated with slow slip in seismogenic regions ( $>$ km) [lde et al., 2007; Peng and Gomberg, 2010] and LPLD (Long Period Long Duration) events observed at decametric scale during hydraulic fracturing operations in a shale-gas reservoir [Das and Zoback, 2013a, 2013b].

Figure 4a shows that the fault activation evolves from an initially aseismic response until the maximum injected pressure of $3.5 \mathrm{MPa}$ (step 15 in Figures 2 and 4a) to the transition to seismic mode. The fault opens in good accordance with the change of effective stress state induced by the injection. The aseismic deformation is characterized by a maximum slip of $0.3 \times 10^{-3} \mathrm{~m}$ associated to an intense opening of $0.5 \times 10^{-3} \mathrm{~m}$. During this period, the dilation of the fault exceeds the fault slip. It corresponds to the period when pressure is increased step by step at the injection point. At the transition to seismic displacement, the deformation mode switches, and slip exceeds twice the dilation as 1.2 and $0.6 \times 10^{-3} \mathrm{~m}$, respectively. This transition occurs at the maximum pressure of $3.5 \mathrm{MPa}$, when some pressure transient drops of $\sim 0.1 \mathrm{MPa}$ are observed.

In order to determine the evolution of stresses on the fault during slip and fluid diffusion associated with injection, we used 3-D hydromechanical modeling. The 3DEC code [Itasca Consulting Group, Inc., 2003] (see supporting information) was employed to represent the fault by a slip plane in an elastic medium $(40 \mathrm{~m} \times 40 \mathrm{~m} \times 60 \mathrm{~m})$ (Figure 4b). For simplicity, fault initial hydraulic and mechanical properties are considered homogeneous. The principal stresses measured in situ at the depth of the experiment are applied to the six boundaries of the model. The field experiment is simulated by imposing at a point 
source (grid point called "injection" in Figure 4b) the time-dependent pressure step increase measured in the injection hole (Figure 2). To simulate variation in frictional resistance during sliding, a friction-weakening law (see supporting information) was employed with a reduction of the friction coefficient from an initial peak value (0.6) to a residual one (0.4) when the frictional resistance exceeded (friction was allowed to decrease over a $0.8 \times 10^{-4} \mathrm{~m}$ critical slip distance representing the roughness of the fault). Fluid pressure gradient and viscous forces associated with fluid flow are represented through the fault only (i.e., the surrounding medium is impervious). Model results reproduce reasonably well the measured fault displacements (Figure 4a). Simulations indicate that, at the onset of the seismicity (step 15), the slipping patch has a diameter of about $30 \mathrm{~m}$, reaching the control point " $\mathrm{M}$ " located $25 \mathrm{~m}$ below the injection point and the seismic sensors (Figure 4b). We did not attempt to make an exact match of simulated and experimental data (indeed, the observed discrepancy is related to the simple homogeneous properties affected to the fault) but rather tried to obtain a reasonable agreement to the general evolution and magnitude of data. Consequently, we focused on identifying the transitions between elastic and inelastic slip, and fault activity after step 15 that covers the most intense period of seismic activity shown in Figure 2 . The model shows that when seismicity occurs, pressurized fluids flow in a relatively large slipping area of the fault characterized by a shear stress change of 0.4 to $1.2 \mathrm{MPa}$ (Figure $4 \mathrm{~b}$ ). At the boundaries of this zone invaded by pressurized fluids, an accumulation of the shear stress occurs, with values comprised between 1.4 and $1.6 \mathrm{MPa}$.

The evolution of the shear and effective normal stresses as a function of fault shear displacement at the injection point and control point $\mathrm{M}$ shows that there is a strong effective normal stress decrease controlled by fluid pressure injection during the initial aseismic period (Figures $4 a$ and $4 c$ ). The effective normal stress drop is lower at point $M$ than at the injection point because of the lower fluid pressure variation at this point located $25 \mathrm{~m}$ below the injection. Before step 14, shear stress initially accumulates at point $M$ with small elastic displacement and while the injected pressure has not reached this point. There is no inelastic slip on the fault at this time because the finite stiffness of the fault produces a nonzero elastic shear displacement. After step 14, a progressive decrease of the shear stress occurs until the fault stops slipping. Seismicity occurs when shear stress drops with magnitude of 0.5 to $0.6 \mathrm{MPa}$. Two competing mechanisms occur during the period of steps 14 and 15: (1) a reduction of effective stress related to the diffusion of increasing fluid pressures from the injection point, and (2) slight increase of shear stress $(0.1$ to $0.3 \mathrm{MPa})$ at the pressure front. Interestingly, at point $\mathrm{M}$ ( $25 \mathrm{~m}$ below injection point), this small shear stress increase is sufficient to exceed the fault strength and promote friction weakening (from 0.6 to 0.4 ). During the seismic period, a slip amplitude of about $0.5 \times 10^{-3} \mathrm{~m}$ can be produced. This slip is associated with a shear stress drop of $0.1 \mathrm{MPa}$ at a distance of several meters ( 25 to $34 \mathrm{~m}$ ) from the injection source, into areas slightly pressurized by injection. Thus, after step 15, the slipping patch may have reached a critical size, large enough to induce a sufficient weakening at its boundaries to control further slip and seismicity during shut-in when changes in fluid pressure are small (step from 15 to 21). The shear stress change thus shows how evolving slip and fluid pressure transfer shear stress to the rupture front, promoting further slip. Meanwhile, within the slipping zone, shear stress drops between 0.1 and $1 \mathrm{MPa}$ are consistent with observations of tectonic earthquakes [Scholz, 2002]. The seismicity induced by this slip might occur directly on the fault or in the surrounding medium. One hypothesis is that the TLS are generated by slow slip on the fault, while the microseismicity occurred on surrounding small fractures to accommodate for the main fault displacement. However, this interpretation should be checked by locating the seismic events. This experiment, therefore, shows different fault seismic responses, from stable, aseismic deformations to slow slip with tremors sequences, as well as to fast, unstable slip with microearthquakes.

All our observations indicate that the observed seismic signals are likely generated by shear motions due to the high-fluid pressure within the fault during hydraulic stimulation. This result is in accordance with other studies on faults that document triggering mechanisms of seismic signals (tremors, microearthquakes, etc.) controlled by high-fluid pressures and associated weakening [Thomas et al., 2009; Shelly, 2015].

\section{Conclusion}

Thanks to a proximal monitoring (meters) at high frequency of a fluid injection directly into a fault, we identified three types of seismic signals: microearthquakes (SE), tremors (TLS), and long period (LP) events, respectively. Our interpretation suggests that the combination of these different signals might be a proxy 
Acknowledgments

This work is financed by the ANR "Captage de $\mathrm{CO}_{2}$ " through the "HPPP- $\mathrm{CO}_{2}$ " project, by the PACA county through the "PETRO PRO" project, and by the ANR "RiskNat" through the "SLAMS" project. The data were obtained and distributed by the working group of the HPPP-CO2 project led by Y. Guglielmi. We thank the engineers team (D. Boyer, A. Cavaillou, and M. Auguste) of the Laboratoire Souterrain à Bas Bruit de Rustrel (LSBB) (http://lsbb.oca.eu/), and the SITES S.A. We also thank the two anonymous reviewers for the constructive comments and suggestions that improved our paper. This work was partially sponsored by ANR "HYDROSEIS" under the contract ANR-13-JS06-0004-01.

The Editor thanks two anonymous reviewers for their assistance in evaluating this paper. to fault hydromechanical evolution to instability. Indeed, microearthquakes combined with tremors might reflect weakening of the fault frictional properties in and at the boundaries of the pressurized patch that we deduced from hydromechanical modeling of experimental data. Our experiment especially shows how coupled high-fluid pressures and fault weakening may drive a fault zone to a complex seismic sequence, highlighting the importance to monitor the diversity of the seismicity in the near field of the source to investigate accurately the slip instability processes.

\section{References}

Amitrano, D., M. Arattano, M. Chiarle, G. Mortara, C. Occhiena, M. Pirulli, and C. Scavia (2010), Microseismic activity analysis for the study of the rupture mechanisms in unstable rock masses, Nat. Hazards Earth Syst. Sci., 10, 831-841.

Becken, M., O. Ritter, P. A. Bedrosian, and U. Weckmann (2011), Correlation between fluids, tremor and creep along the central San Andreas fault, Nature, 480, 87-92.

Bouchon, M., H. Karabulut, M. Aktar, S. Ozalaybey, J. Schmittbuhl, and M. P. Bouin (2011), Extended nucleation of the 1999 Mw 7.6 Izmit earthquake, Science, 331, 877-880.

Chouet, B. (2003), Volcano seismology, Pure Appl. Geophys., 160, 739-768.

Cornet, F. H. (2012), The relationship between seismic and aseismic motions induced by forced fluid injections, Hydrogeol. J., 20, $1463-1466$.

Cornet, F. H., J. Helm, H. Poitrenaud, and A. Etchecopar (1997), Seismic and aseismic slips induced by large-scale fluid injections, Pageoph, $150,563-583$

Das, I., and M. D. Zoback (2011), Long-period, long-duration seismic events during hydraulic fracture stimulation of a shale gas reservoir, The Leading Edge, 30, 778-786, doi:10.1190/1.3609093.

Das, I., and M. D. Zoback (2013a), Long-period long-duration seismic events during hydraulic stimulation of shale and tight gas reservoirPart 1: Waveform characteristics, Geophysics, 78, doi:10.1190/GEO2013-0164.1.

Das, I., and M. D. Zoback (2013b), Long-period long-duration seismic events during hydraulic stimulation of shale and tight gas reservoirPart 2: Location and mechanisms, Geophysics, 78, doi:10.1190/GEO2013-0165.1.

Derode, B., F. Cappa, Y. Guglielmi, and J. Rutqvist (2013), Coupled seismo-hydromechanical monitoring of inelastic effects on injectioninduced fracture permeability, Int. J. Rock Mech. Min. Sci., 61, 266-274, doi:10.1016/j.jirmms.2013.03.008.

Guglielmi, Y., F. Cappa, and D. Amitrano (2008), High-definition analysis of fluid-induced seismicity related to the mesoscale hydromechanical properties of a fault zone, Geophys. Res. Lett., 35, L06306, doi:10.1029/2007GL033087.

Guglielmi, Y., F. Cappa, H. Lançon, R. Janowczyk, C. F. Tsang, and J. S. Y. Wang (2014), ISRM suggested method for step-rate injection method for fracture in-situ properties (SIMFIP): Using a 3-components borehole deformation sensor, Rock Mech. Rock Eng., 47, 303-311, doi:10.1007/s00603-013-0517-1.

Ide, S., D. R. Shelly, and G. C. Beroza (2007), Mechanism of deep low frequency earthquakes: Further evidence that deep nonvolcanic tremor is generated by shear slip on the plate interface, Geophys. Res. Lett., 34, L03308, doi:10.1029/2006GL028890.

Itasca Consulting Group, Inc. (2003), 3DEC 3-Dimensional Distinct Element Code, ICG, Minneapolis, Minn.

Jeanne, P., Y. Guglielmi, and F. Cappa (2012), Multiscale seismic signature of a small fault zone in carbonate reservoir: Relationships between $V_{P}$ imaging, fault zone architecture and cohesion, Tectonophysics, 554-557, 185-201, doi:10.1016/j.tecto.2012.05.012.

Miyazawa, M., and E. E. Brodsky (2008), Deep low-frequency tremor that correlates with passing surface waves, J. Geophys. Res., 113, B01307, doi:10.1029/2006JB004890.

Muir-Wood, R. M., and G. C. P. King (1993), Hydrological signatures of earthquake strain, J. Geophys. Res., 98, 22,035-22,068, doi:10.1029/ 93JB02219.

Obara, K. (2002), Non-volcanic deep tremor associated with subduction in southwest Japan, Science, 296, 1679-1681, doi:10.1126/ science.1070378.

Peng, Z., and J. Gomberg (2010), An integrated perspective of the continuum between earthquakes and slow-slip phenomena, Nat. Geosci., doi:10.1038/BGEO940.

Scholz, C. H. (2002), The Mechanics of Earthquakes and Faulting, Cambridge Univ. Press, Cambridge.

Shelly, D. R. (2015), Complexity of the deep San Andreas Fault zone defined by cascading tremor, Nat. Geosci., doi:10.1038/NGEO2335.

Shelly, D. R., G. C. Beroza, S. Ide, and S. Nakamula (2006), Low-frequency earthquakes in Shikoku, Japan, and their relationship to episodic tremor and slip, Nature, 442, 188-191, doi:10.1038/nature04931.

Sibson, R. H. (1982), Fault zone models, heat flow, and the depth distribution of earthquakes in the continental crust of the United States, Bull. Seismol., 72, 151-163.

Tary, J. B., M. van der Baan, and D. W. Eaton (2014), Interpretation of resonance frequencies recorded during hydraulic fracturing treatments, J. Geophys. Res. Solid Earth, 119, 1295-1315, doi:10.1002/2013JB010904.

Thomas, A. M., R. M. Nadeau, and R. Burgmann (2009), Tremor-tide correlations and near-lithostatic pore pressure on the deep San Andreas fault, Nature, 462, 1048-1051, doi:10.1038/nature08654.

Zigone, D., C. Voisin, E. Larose, F. Renard, and M. Campillo (2011), Slip acceleration generates seismic tremor like signals in friction experiments, Geophys. Res. Lett., 38, L01315, doi:10.1029/2010GL045603. 